\title{
Discrete survey of 5-cm OH emission from planetary and proto-planetary nebulae
}

\author{
J.-F. Desmurs ${ }^{1}$, A. Baudry ${ }^{2}$, P. Sivagnanam ${ }^{3}$, C. Henkel ${ }^{4}$ \\ and A. M. S. Richards ${ }^{5}$ \\ ${ }^{1}$ Observatorio Astronómico Nacional, Madrid, Spain \\ email: desmurs@oan.es \\ ${ }^{2}$ LAB - Observatoire Aquitain des Sciences de l'Univers, Floirac, France \\ ${ }^{3}$ Observatoire de Paris, France \\ ${ }^{4}$ MPIfR, Bonn, Germany \\ ${ }^{5}$ Jodrell Bank Observatory, UK
}

\begin{abstract}
We have undertaken a comprehensive search for the first excited state of $\mathrm{OH}$ emission $\left(\mathrm{J}=5 / 2,{ }^{2} \Pi_{3 / 2}\right)$ in Planetary and Proto- Planetary Nebulae. With the Effelsberg telescope, we confirm the detection of $\mathrm{Vy} 2-2$ and we present one new detection in the pPN K3-35. This detection has been confirmed by subsequent observations made at $6035 \mathrm{MHz}$ with the MERLIN interferometer. This is the first detection of $6 \mathrm{GHz} \mathrm{OH}$ maser emission from a post-AGB star.
\end{abstract}

Keywords. Maser, stars: AGB and post-AGB, radio lines: stars

\section{Introduction}

PNe evolve from the envelopes of AGB stars, through the very short ( $1000 \mathrm{yr})$ phase of protoplanetary nebula $(\mathrm{pPN})$. During this phase, the nebular morphology and kinematics are dramatically altered: the spherical, slowly expanding AGB envelope becomes a PN with, usually, axial symmetry and high axial velocities. Sensitive observations of Planetary Nebulae in the $5 \mathrm{~cm}$ lines of $\mathrm{OH}$ are motivated by several reasons. The $6 \mathrm{GHz}$ observations probe highly excited inner layers and provide important information; excitation models are complex and still uncertain, and predictions on the main pumping routes leading to $\mathrm{OH}$ inversion of the ground state will be constrained by observations of the $J=5 / 2$ state.

\section{Results and discussion}

With the $100 \mathrm{~m}$ MPIfR radiotelescope at Effelsberg, we have undertaken a complete discrete source survey for the first excited state of $\mathrm{OH}$ emission $\left(J=5 / 2,{ }^{2} \Pi_{3 / 2}\right)$ in 31 $\mathrm{PN}$ and pPN above a declination of -20 degrees. It is based on previous $18 \mathrm{~cm} \mathrm{OH}$ surveys in which we have selected all sources exhibiting both 1612 and/or 1665/1667 $\mathrm{MHz}$ emission. We reached an average noise value of about $3 \sigma \sim 30 \mathrm{mJy}$ for a spectral resolution of $0.29 \mathrm{~km} / \mathrm{s}$. We confirm the detection of Vy 2-2 (see Desmurs et al. 2002) and we detected one new source, the young PN K 3-35, as a single $1 \mathrm{~km} / \mathrm{s}$-wide component around $21 \mathrm{~km} / \mathrm{s}$ LSR velocity. This is the first detection of $6 \mathrm{GHz} \mathrm{OH}$ maser emission from a post-AGB star.

To confirm the $6035 \mathrm{MHz}$ maser emission detected with Effelsberg in $\mathrm{K} 3-35$, we made new observations using the MERLIN interferometer in the ground state $\left({ }^{2} \Pi_{3 / 2}, J=3 / 2\right)$ and the first excited level $\left({ }^{2} \Pi_{3 / 2}, J=5 / 2\right)$. Figure 1 presents our map of the $6035 \mathrm{MHz}$ 


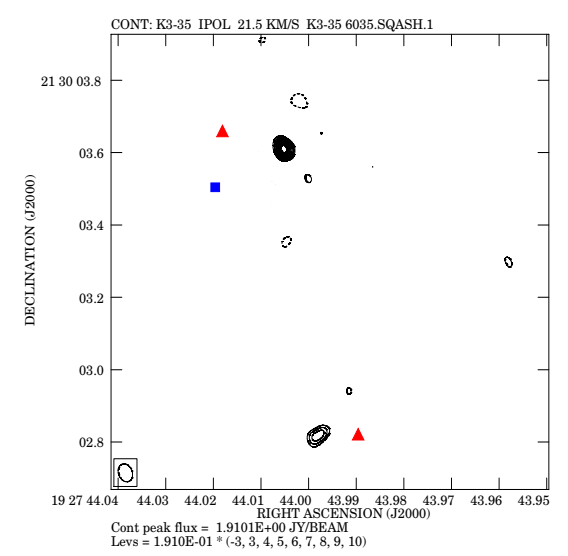

Figure 1. Map of the $\mathrm{OH} 6035 \mathrm{MHz}$ maser distribution (line contours) compared to the 1612 (blue square) and $1720 \mathrm{MHz}$ (red triangle) maser emission centers.

maser emission (see line contours). The 18-cm positions (position uncertainty is about 40 mas) derived from our interferometric observations are shown for the $1720 \mathrm{MHz}$ (red triangle) and the $1612 \mathrm{MHz}$ (blue square). Other 18-cm OH components detected with the Nançay radio telescope around 9 and $18 \mathrm{~km} / \mathrm{s}$ did not show any $6 \mathrm{GHz}$ counterpart or $18-\mathrm{cm}$ compact emission in the MERLIN data.

$\mathrm{K} 3-35$, is considered to be a very young PN (see OH maser observations by Zijlstra et al. 1989) situated in the constellation Vulpecula. VLA observations by Miranda et al. (2001) at $22 \mathrm{GHz}$ reveal the shape of a bipolar structure typical of Proto Planetary Nebulae and allow the authors to conclude that this object has just begun its transformation into a planetary nebula. K 3-35 has been detected at $18 \mathrm{~cm}$ in the 1612, 1667, and $1720 \mathrm{MHz}$ OH lines (Engels et al. 1985, te Linkel Hekkert 1990, Gómez et al. 2006). Our interferometric observations of K $3-35$, show the quasi spatial coincidence of the $18 \mathrm{~cm}$ and $5 \mathrm{~cm}$ maser emissions.

The absence of detectable excited emission at $5 \mathrm{~cm}$ in pPNe (except in $\mathrm{Vy} 2-2$ and $\mathrm{K} 3-35$ ) tends to argue in favour of a pumping scheme based on the absorption of 35 and $53 \mu \mathrm{m}$ photons. In the case of $\mathrm{Vy} 2-2$ the ionisation shell from where the maser emission seems to originate, may present physical conditions (shock, higher temperature and density) similar to those prevailing in HII regions. We note that $1720 \mathrm{MHz}$ masers are exceptionally rare around evolved stars (but have been observed in many star forming regions). A hybrid pumping model applying to both OH/IR stars and HII regions or even a pumping scheme similar to $\mathrm{OH}$ maser emission in massive star forming regions may be successful.

\section{References}

Desmurs, J.-F., Baudry, A., Sivagnanam, P., \& Henkel, C. 2002, A $\& A$ 394, 975

Engels, D., Schmid-Burgk, J., Walmsley, C. M., \& Winnberg, A. 1985, A\&A A 148, 344

Gómez, Y., Tafoya, D., Anglada, G., Franco-Hernandez, R., Torrelles, J. M., \& Miranda, L. F. $2006 R M x A C 26,22$

Miranda, L. F., Gómez, Y. Anglada, G., \& Torrelles, J. M. 2001 Nature 414, 284

te Linkel Hekkert 1990, PhD Thesis

Zijlstra, A. A., te Linkel Hekkert, P., Pottasch, S. R., Caswell, J. L., Ratag, M., \& Habing H. J., 1989, A\&A 217, 157 